\title{
Iatrogenic profound hypoalphalipoproteinaemia: an unrecognised cause of very low HDL cholesterol
}

\author{
Michael J Murphy, Andrew Duncan, Barry D Vallance, Christopher J Packard, \\ Denis St J O'Reilly
}

\begin{abstract}
Summary
A significant reduction in plasma high density lipoprotein (HDL) cholesterol is a recognised consequence of treatment with probucol. By contrast, fibrate therapy in general has the opposite effect. We report two cases where the combination of probucol and a fibrate led to profoundly reduced plasma levels of HDL cholesterol associated with very low levels of apolipoprotein A-I (apoA-I). In the first, bezafibrate was added to probucol, and in the second, probucol added to a combination of simvastatin and fenofibrate. In both cases, plasma levels of HDL and apoA-I returned towards normal after discontinuation of one or both drugs, indicating that the reduction was reversible.
\end{abstract}

Keywords: probucol, fibrates, hypoalphalipoproteinaemia

\section{Case 1}

A 57-year-old woman was referred to the cardiovascular risk factor clinic with hypercholesterolaemia (total cholesterol $9.35 \mathrm{mmol} /$ 1). She had no other prominent risk factors for cardiovascular disease and physical examination revealed no signs of hyperlipidaemia. For several years previously she had suffered from rheumatoid arthritis and was being treated with non-steroidal anti-inflammatory drugs. Baseline investigations included biochemical evaluation of thyroid, liver and renal function as well as fasting blood glucose and full blood count; no abnormalities were detected. Despite dietary intervention, total cholesterol remained

Institute of Biochemistry, Royal Infirmary, Glasgow G4 OSF, UK

MJ Murphy

A Duncan

CJ Packard

D St J O'Reilly

Hairmyres Hospital, Eaglesham Road, East Kilbride, UK BD Vallance stopping bezafibrate HDL cholesterol had returned to $0.7 \mathrm{mmol} / 1$ and apoA-I to $0.79 \mathrm{~g} / 1$. HDL rose further after probucol was discontinued. Cumulative results are shown in figure 1.

\section{Case 2}

An obese 36-year-old man with severe hypercholesterolaemia (total cholesterol $11.5 \mathrm{mmol} /$ 1; LDL cholesterol $8.55 \mathrm{mmol} / \mathrm{l}$ ) and tendon xanthomata underwent coronary artery bypass grafting following recovery from a myocardial infarct. Investigations of thyroid, liver and renal function were carried out in addition to fasting blood glucose and full blood count; no causes of secondary hyperlipidaemia were identified. Over a period of several years his hypercholesterolaemia was treated with various lipid-lowering agents, either alone or in combination; they included colestipol, nicotinic acid, gemfibrozil and subsequently simvastatin, fenofibrate and probucol. While on treatment, the patient sustained a second myocardial infarct and underwent repeat bypass grafting. When probucol $500 \mathrm{mg}$ bid was added to a combination of simvastatin $40 \mathrm{mg}$ nocte and fenofibrate $200 \mathrm{mg}$ tid, plasma HDL cholesterol fell within three months to $0.15 \mathrm{mmol} / \mathrm{l}$ (normal $>1.0 \mathrm{mmol} / \mathrm{l}$ ); apoA-I at

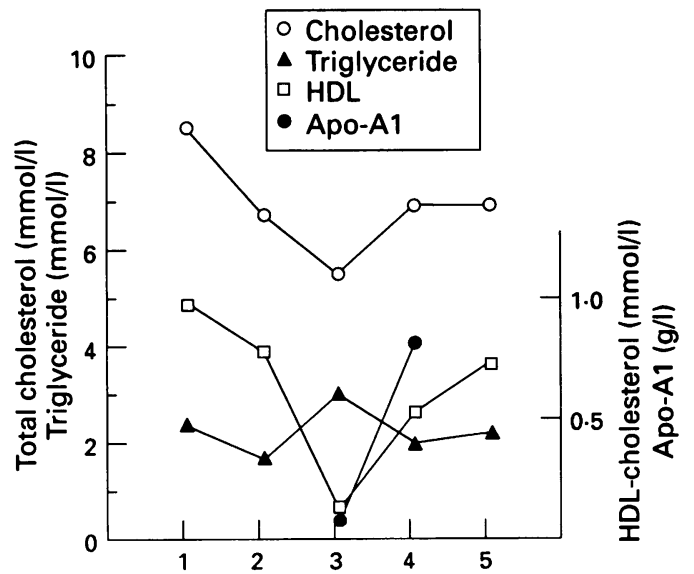

Figure 1 Data points: 1 , probucol $500 \mathrm{mg}$ bid started; 2 , bezafibrate $400 \mathrm{mg}$ nocte started; 3 , bezafibrate stopped; 4 , probucol stopped; 5 , measurements repeated off lipid-lowering treatment. Time intervals vary between data points shown. See text for details probucol (normal $>1.0 \mathrm{mmol} / \mathrm{l}$ ). Three months after the introduction of bezafibrate, HDL cholesterol had fallen to $0.1 \mathrm{mmol} / \mathrm{l}$ at which point the apoA-I level was $0.06 \mathrm{~g} / 1$ (reference range $0.7-1.3 \mathrm{~g} / \mathrm{l})$. Within three months of 
this time was $0.15 \mathrm{~g} / \mathrm{l}$ (reference range 0.7-1.3 g/1). Before the introduction of probucol, plasma HDL had ranged between 0.75 and $1.35 \mathrm{mmol} / \mathrm{l}$. All lipid-lowering agents were discontinued and measurements of plasma HDL and apoA-I repeated four weeks later. Both had returned to normal (HDL was $1.1 \mathrm{mmol} / \mathrm{l}$ and apoA-I $1.15 \mathrm{mmol} / \mathrm{l}$ ). Cumulative results are shown in figure 2 .

\section{Discussion}

The basis for the reduction in HDL cholesterol normally seen with probucol probably relates to a decrease in the synthesis of apolipoprotein A-I (apoA-I), one of the principal protein components of HDL particles. ${ }^{1}$ However, there is evidence that it may be due in part to an increase in the plasma concentration and activity of cholesteryl ester transfer protein. ${ }^{2}$ Although decreased lipoprotein lipase activity has been reported during probucol treatment, ${ }^{3}$ very low density lipoprotein (VLDL) catabolism does not appear to be affected. ${ }^{4}$ In contrast with probucol, bezafibrate is associated with concomitant rises in HDL cholesterol, apoA-I and apoA-II, both in patients with hypertriglyceridaemia ${ }^{5}$ and those with hypercholesterolaemia. ${ }^{6}$ Increased plasma apoA-I levels have also been described with fenofibrate, ${ }^{7}$ usually in association with a rise in plasma HDL cholesterol ${ }^{8}$ (see table).

The profound reduction in plasma levels of apoA-I and HDL seen in case 1 soon after bezafibrate was added to probucol made an interaction between the two drugs likely. This

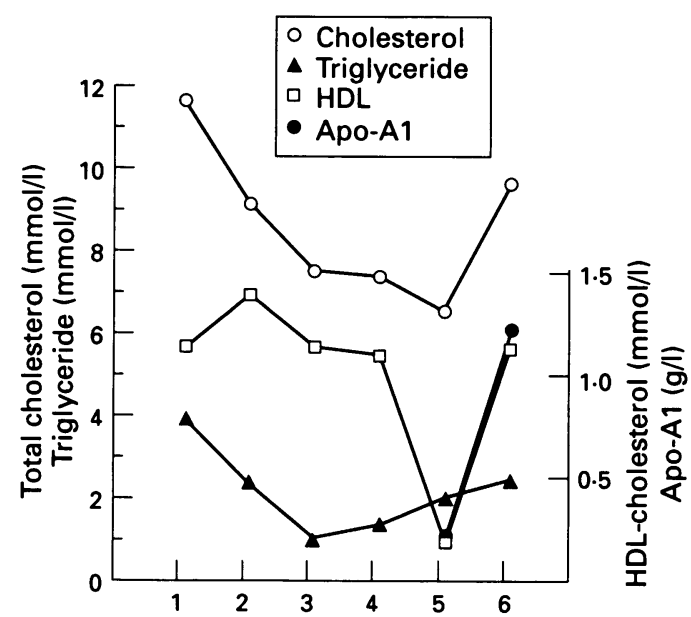

Figure 2 Data points: 1, simvastatin $10 \mathrm{mg}$ nocte started; 2 , simvastin $40 \mathrm{mg}$ nocte; fenofibrate $100 \mathrm{mg}$ tid started; 3 , fenofibrate increased to $200 \mathrm{mg}$ tid; 4 , probucol $500 \mathrm{mg}$ bid added; 5, all lipid-lowering drugs stopped; 6 , measurements repeated off lipid-lowering treatment. Time intervals vary between data points shown. See text for details. suspicion was strengthened when levels of both rose once bezafibrate was discontinued, returning in the case of apoA-I to normal and in the case of HDL to values similar to those seen prior to the introduction of bezafibrate (see figure 1). Case 2 was being treated with both fenofibrate and simvastatin when probucol was added, so the possibility of an interaction involving simvastatin cannot be ruled out. However, the development of profoundly reduced plasma levels of HDL cholesterol and apoA-I soon after the advent of another probucol-fibrate combination seems unlikely to have been coincidental, and an interaction between probucol and fenofibrate is therefore more likely.

The exact nature of this interaction between probucol and fibrates remains speculative. Potentiation of probucol by the fibrates seems more likely, a priori, than alteration of the properties of bezafibrate and fenofibrate by probucol. Fibrate-induced changes in the structure and composition of HDL particles may have rendered them more susceptible to the effects of probucol on apoA-I synthesis, or may have made them more unstable, thereby resulting in an increased fractional catabolic rate. Alternatively, displacement of probucol by fibrates from binding sites on plasma proteins may have enhanced the bioavailability of probucol, thereby potentiating its actions on HDL synthesis and/or breakdown. Alterations in the absorption and/or excretion of probucol induced by the fibrates are less plausible explanations.

Further characterisation of this interaction will require detailed examination of sequential fasting lipid profiles in each patient, initially off and subsequently on treatment with the agents involved, separately and in combination. (Wash-out periods between profiles would need to take account of the long half-life of probucol in the body.) Studies might usefully include analysis of the associated changes in HDL composition and subclasses, as well as turnover studies of apoA-I synthesis. As the patients were given different fibrates, it would in addition be of interest to compare the results obtained in each patient with bezafibrate and fenofibrate.

The possibility of inducing profound hypoalphalipoproteinaemia, and a commensurate reduction in plasma HDL cholesterol, should be borne in mind if probucol is to be combined with fibrate therapy. Although in both of these cases the alteration in lipoprotein metabolism reversed following discontinuation of the combination, it cannot be assumed that this will always occur. Finally, the potential effects on cardiovascular risk of iatrogenic profound hypoalphalipoproteinaemia are unknown.
Table Effect of drug treatment on lipid levels

\begin{tabular}{lllll}
\hline Drug & Total cholesterol & LDL cholesterol & HDL cholesterol & Triglycerides \\
\hline Probucol & $\downarrow 5-20 \%$ & $\downarrow 10-15 \%$ & $\downarrow 20-25 \%$ & $\leftrightarrow$ \\
Fibrates & $\downarrow 15-30 \%$ & $\downarrow 5-35 \%$ & $\uparrow 10-30 \%$ & $\downarrow 40-50 \%$ \\
\hline
\end{tabular}

\section{Learning point}

Prescribers should be aware that combining probucol with a fibrate may result in very low levels of $\mathrm{HDL}$ 
1 Nestel PJ, Billington T. Effects of probucol on low density lipoprotein removal and high density lipoprotein synthesis. Atherosclerosis 1981; 38: 203-9.

2 McPherson R, Hogue M, Milne RW, Tall AR, Marcel YL Increase in plasma cholesteryl ester transfer protein during Increase in plasma cholesteryl ester transfer protein during

3 Miettinen TA, Huttunen JW, Kuusi T, et al. Effect of probucol on the activity of postheparin plasma liport probucol on the activity of postheparin plasma lipoprotein lipase and hepatic lipase. Clin Chim Acta 1981; 113: 59-64. Lock DR, Kuisk I, Gonen B, Patsch W, Schonfeld G. Effect of probucol on the composition of lipoproteins and on VLDL

5 Vessby E, Lithell H, Hellsing K, et al. Efiects of bezafibrate Vessby E, Lithell $\mathrm{H}$, Hellsing $\mathrm{K}$, et al. Efiects of bezafibrate
on the serum lipoprotein lipid and apolipoprotein composition, lipoprotein triglyceride removal capacity and the fatty acid composition of the plasma lipid esters. Atherosclerosis 1980; 37: 257-69.
6 Mordasini R, Riesen W, Oster P, Keller M, Middelhoff G, Lang PD. Reduced LDL- and increased HDL-apoprotein in patients with hypercholesterolaemia under treatment with bezafibrate. Atherosclerosis 1981; 40: 153-8.

7 Micheli M, Pometta D, Gustafsson A. Treatment of hyperlipoproteinemia type IIA with a new phenoxy-isobutyric acid derivative, procetofen. Int $\mathcal{f}$ Clin Pharmacol Biopharm $1979 ; 17: 503-6$.

8 Lehtonen A, Viikari J. Fenofibrate and cholestyramine in type II hyperlipoproteinemia. Artery 1982; 10: 353-67.

\title{
Polymyalgia rheumatica, temporal arteritis and malignancy
}

\author{
CA Speed, I Haslock
}

\begin{abstract}
Summary
The use of steroid therapy in polymyalgia rheumatica and temporal arteritis is necessary and usually effective, but may mask coexisting disease. The importance of early consideration of other disorders in such patients is illustrated by three case histories.
\end{abstract}

Keywords: polymyalgia rheumatica, temporal arteritis, malignancy, Waldenstrom's macroglobulinaemia

Polymyalgia rheumatica (PMR) and temporal arteritis (TA) are disorders which are usually steroid responsive and easily monitored by the clinical picture and acute phase parameters such as the erythrocyte sedimentation rate (ESR). The clinical features are often nonspecific and steroid therapy may mask features of coexisting diseases. We describe three cases of PMR/TA, in which an apparent flare of the disease was in fact an initial presentation of another disease - in two cases, a malignancy. The importance of maintaining a high degree of clinical suspicion in the management of such patients is emphasised and the association of $\mathrm{PMR} / \mathrm{TA}$ with other disorders is discussed.

\section{Case histories}

Case 1

A 60-year-old male smoker presented with a two-month history of bilateral temporal headaches. Examination revealed pulsatile, thickened temporal arteries. An ESR was $97 \mathrm{~mm} / \mathrm{h}$; a temporal artery biopsy confirmed cranial arteritis. A chest X-ray performed at the time was consistent with chronic obstructive airways disease, with no focal lesions. His symptoms and ESR responded dramatically to oral steroids.

Eighteen months later, he was re-referred by his general practitioner with letheragy and an ESR of $84 \mathrm{~mm} / \mathrm{h}$. This had failed to respond to increasing his steroids from 5 to $40 \mathrm{mg}$ daily. A right supraclavicular lymph node was palpable. A chest X-ray revealed an enlarged right hilum and right lower lobe collapse. A lymph node biopsy confirmed adenocarcinoma.

Case 2

A 60-year-old male smoker presented to his general practitioner with a seven-month history of temporal headaches, scalp tenderness and proximal myalgia. An ESR was $116 \mathrm{~mm} / \mathrm{h}$. A diagnosis of temporal arteritis was made and he responded dramatically to steroids. One month later, whilst on $40 \mathrm{mg}$ prednisolone daily, he returned to his doctor complaining of lethargy. An ESR was $107 \mathrm{~mm} / \mathrm{h}$. He failed to respond to increasing his steroid dosage and was referred to the rheumatology clinic. A chest X-ray was performed and revealed consolidation and collapse of the right lower and middle lobes. B:onchoscopy confirmed squamous cell carcinoma.

\section{Case 3}

A 53-year-old woman presented to her general practitioner with a two-month history of proximal muscle stiffness and non-specific head- N aches. Examination was unremarkable; an ESR श्? was $65 \mathrm{~mm} / \mathrm{h}$. A diagnosis of polymyalgia rheumatica was made. Her symptoms responded to prednisolone $(30 \mathrm{mg} /$ day) within two days. However, her ESR remained elevated, varying between 45 and $62 \mathrm{~mm} / \mathrm{h}$, so she was referred for a specialist opinion. Further investigations revealed an IgM kappa paraproteinaemia; urinary Bence Jones proteins were absent. Her plasma viscosity was $1.89 \mathrm{cp}$; full blood count and C-reactive protein were normal, apart from a mild neutrophilia. A bone marrow biopsy was consistent with a diagnosis of Waldenstrom's macro-
Department of Cleveland Hospital, Middlesbrough Cleveland TS4 3BW, CA Speed

I Haslock 